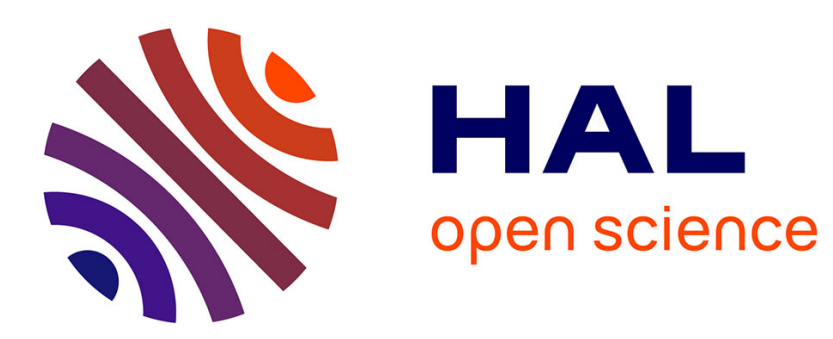

\title{
Nonlinear operators on graphs via stacks
}

\author{
Santiago Velasco-Forero, Jesus Angulo
}

\section{To cite this version:}

Santiago Velasco-Forero, Jesus Angulo. Nonlinear operators on graphs via stacks. Geometric Science of Information, Oct 2015, Palaiseau, France. pp.654-663, 10.1007/978-3-319-25040-3_70 . hal01110976

\section{HAL Id: hal-01110976 \\ https://hal.science/hal-01110976}

Submitted on 29 Jan 2015

HAL is a multi-disciplinary open access archive for the deposit and dissemination of scientific research documents, whether they are published or not. The documents may come from teaching and research institutions in France or abroad, or from public or private research centers.
L'archive ouverte pluridisciplinaire HAL, est destinée au dépôt et à la diffusion de documents scientifiques de niveau recherche, publiés ou non, émanant des établissements d'enseignement et de recherche français ou étrangers, des laboratoires publics ou privés. 


\title{
Nonlinear operators on graphs via stacks
}

\author{
Santiago Velasco-Forero Jesús Angulo \\ MINES ParisTech \\ PSL-Research University \\ Centre de Morphologie Mathématique
}

\begin{abstract}
We consider a framework for nonlinear operators on functions evaluated on graphs via stacks of level sets. We investigate family of transformations which includes adaptive flat and non-flat erosions and dilations. Additionally, we note the connection to mean motion curvature on graphs. Proposed operators are illustrated in the cases of functions on graphs, textured meshes and graphs of images.
\end{abstract}

\section{Introduction}

Recent years have witnessed an enormous growth of interest in the description and analysis of problems via similarities or dependencies between data elements. A common way to represent this structure is to use graphs, so that data elements are indexed by graph nodes, and the strength of dependences between pairs of elements is represented by corresponding weighted graph edges. In this paper, we analyse nonlinear (morphological) operators in the context of discrete signal processing on graphs 1 . Our framework is used to extend the traditional adaptive (non-flat) morphology on images to more complex structures as sets of images, meshes, point clouds [2] and so on. In graph-based modelling, digital images are a particular case, where the pixel information is represented by the two-dimensional rectangular grid, and pixels correspond to graph nodes related by links according to the four or eight adjacent neighbourhood.

On the one hand, we note that in the literature, one can find some works about nonlinear filters on graphs and hypergraphs, particularly mathematical morphology operators in the algebraic sense [3|4|5|6|7|8|9], where the couple of nonlinear operator (dilation/erosion) are maps from two different lattices, i.e., they are maps "from nodes to edges" or "edges to nodes". On the other hand, some regularisation techniques and nonlinear operators have been introduced on function evaluated on graph via directional derivative 10, 11 [12 or discrete version of the $p$-Laplacian 13 . We adopt a different viewpoint, our approach is inspired from the signal processing approach on graphs [14]15]16[17]. Thus, we firstly review graph signal decomposition by upper-level sets, convolution and diffusion on graphs, and then we present a general formulation of flat and non-flat morphology on graphs, a family of nonlinear transformations and its connection to mean curvature motion on graphs [18/17/19]. Finally, the experimental section includes some examples to illustrate the interest of our method. 


\section{Convolution and morphology by stacks on graphs}

\section{$2.1 \quad$ Notation}

We start by introducing the notation used throughout this paper. The objects under study are consider as the nodes of the graph $\mathcal{G}$. A simple, connected, undirected, and weighted graph $\mathcal{G}=(\mathcal{V}, E)$ consists of a set of nodes $\mathcal{V}=\{1,2, \ldots, N\}$ and edges $E=\left\{\left(n, m, w_{n m}\right)\right\}, n, m \in \mathcal{V}$, where $\left(n, m, w_{n m}\right)$ denoted an edge of weight $w_{n m}$ between node $n$ and $m$. The degree $d_{n}$ of a node $n$ is the sum of the edge-weights connected to node $n$, and the degree matrix of the graph consists of degrees of all nodes arranged in a diagonal ma$\operatorname{trix} \mathbf{D}=\operatorname{diag}\left\{d_{1}, d_{2}, \ldots, d_{N}\right\}$. Denote the maximal and minimal degrees by $d_{+}=\max _{i \in \mathcal{V}} d_{i}$ and $d_{-}=\min _{i \in \mathcal{V}} d_{i}$. The adjacency matrix $\mathbf{W}$ of the graph is an $N \times N$ matrix with $\mathbf{W}(n, m)=w_{n m}$, the combinatorial Laplacian matrix is $\mathbf{L}=\mathbf{D}-\mathbf{W}$ and the graph Laplacian $\mathcal{L}=\mathbf{I}-\mathbf{D}^{-1 / 2} \mathbf{W D}^{-1 / 2}$ is a generalisations of the Laplacian on the grid, where frequency and smoothness are relative to $\mathbf{W}$ and interrelated though these operators [20. A graph signal is defined as a scalar valued discrete mapping $\mathbf{f}: \mathcal{V} \rightarrow \mathbb{R}$, such that $\mathbf{f}(n)$ is the value of the signal on node $n$. Thus a graph signal can also be represented as a vector $\mathbf{f}$ in the space of functions from $\mathcal{V}$ to $\mathbb{R}$, denoted by $\mathrm{V}$, with indices corresponding to the nodes in the graph. Additionally, we often analyse operators transforming signals evaluated on graphs, for instance $\phi: \mathbf{f} \rightarrow \phi(\mathbf{f})$, in this case we say that $\phi \in \mathrm{V} \times \mathrm{V}$. Finally, the graph Fourier transform $\hat{\mathbf{f}}$ of a function $\mathbf{f} \in \mathrm{V}$ is the expansion of $\mathbf{f}$ in terms of the eigenvectors of the graph Laplacian, denoted by $\Lambda_{l}$, with $l=1, \cdots, N$. More precisely, it is defined by $\hat{\mathbf{f}}(l):=\left\langle\mathbf{f}, \Lambda_{l}\right\rangle=\sum_{n=1}^{N} \Lambda_{l}^{*}(n) \mathbf{f}(n)$, where according to [14 adopts the conjugate-linear in the definition 1

Definition 1. The upper level set (ULS) of $\mathbf{f} \in \mathrm{V}$ with level $\lambda \in \mathbb{R}$ is defined by

$$
\chi(\mathbf{f}, \lambda)=\{v \in \mathcal{V}: \mathbf{f}(v) \geq \lambda\} .
$$

The set of ULS constitutes a family of decreasing sets: $\lambda \geq \mu \Rightarrow \chi(\mathbf{f}, \lambda) \subseteq \chi(\mathbf{f}, \mu)$ and $\chi(\mathbf{f}, \lambda)=\cap\{\chi(\mathbf{f}, \mu), \mu<\lambda\}$. Any graph signal $\mathbf{f} \in \mathrm{V}$ can be viewed as a unique stack of its cross-sections, which leads to the following superposition description.

Definition 2. The threshold-max superposition of $\mathbf{f} \in \mathrm{V}$ is defined by:

$$
\mathbf{f}(n)=\bigvee_{\lambda \in \mathbb{R}}\{n \in \chi(\mathbf{f}, \lambda)\}
$$

Similar to the image description as a topographic surface in [212223, we consider here the alternative stacking reconstruction using a numerical sum of the characteristic function of upper level sets.

\footnotetext{
${ }^{1}$ For a given matrix $\mathbf{W}=[\mathbf{W}(i, j)]$, the conjugate of $\mathbf{W}$ to be $\mathbf{W}^{*}=[-\mathbf{W}(j, i)]$, i.e., $\mathbf{W}^{*}$ is derived from $\mathbf{W}$ by transposing and negating.
} 
Definition 3. The threshold-linear superposition of $\mathbf{f} \in \mathrm{V}$ is defined by:

$$
\mathbf{f}(n)=\int_{0}^{+\infty} \chi(\mathbf{f}, \lambda)(n) d \lambda
$$

In the particular case of discrete range, $\mathcal{T}=\left\{c_{1}, c_{2}, \ldots, c_{|\mathcal{T}|}\right\}$, the signal $\mathbf{f}$ can be reconstructed from the discrete stack $\chi(\mathbf{f}, \lambda)$ via addition, i.e., $\mathbf{f}(n)=$ $\sum_{\lambda \in \mathcal{T}} \chi(\mathbf{f}, \lambda)(n)$.

Definition 4. We shall say that an operator $\phi \in \mathrm{V} \times \mathrm{V}$ commutes with thresholding if

$$
\phi(\chi(\mathbf{f}, \lambda))=\chi(\phi(\mathbf{f}), \lambda)
$$

for any signal $\mathbf{f} \in \mathrm{V}$ and any value $\lambda \in \mathbb{R}$.

In other words, if an operator $\phi$ commutes with thresholding, processing by $\phi$ the upper level set at $\lambda$ gives the same result as processing first by $\phi$ the signal $\mathbf{f}$ and then thresholding $\phi(\mathbf{f})$ at level $\lambda$.

Definition 5. We shall say that an operator $\phi$ obeys the threshold-linear superposition provided that

$$
\phi(\mathbf{f})=\int_{0}^{+\infty} \phi(\chi(\mathbf{f}, \lambda)) d \lambda
$$

for any signal $\mathbf{f} \in \mathcal{V} \times \mathbb{R}^{+}$. Respectively, $\sum_{\lambda \in \mathcal{T}^{+}} \phi(\chi(\mathbf{f}, \lambda))$ for positive discrete signals.

As it was pointed out in [23], for grey scale images, the threshold-max superposition in (1) is more general than the thresholded-sum superposition in (4) since the latter applies only to nonnegative input signals, while the former applies to any real-valued input signal. But alternatively, the max-superposition restricts the class of operators since it requires the result of $\phi(\chi(\mathbf{f}, \lambda))$ that are binary signals, an assumption not needed by the sum-superposition. In fact, the threshold sum/integral ties well also with linear systems. In our case, we assume that $\mathbf{f} \in \mathcal{V} \times \mathbb{R}^{+}$is continuous and nonnegative so we will consider the reconstruction formula given by (4).

Proposition 1. The class of operators $\phi \in \mathrm{V} \times \mathrm{V}$ that obey the threshold-linear superposition: a) is closed under minimum, maximum and composition. b) It forms a vector space over the field of real numbers under vector addition $\left(\phi_{1}+\right.$ $\left.\phi_{2}\right)(\mathbf{f}):=\phi_{1}(\mathbf{f})+\phi_{2}(\mathbf{f})$ and the scalar multiplication $(c \phi)(\mathbf{f}):=c \phi(\mathbf{f})$ with $c \in \mathbb{R}$.

\subsection{Convolution on graphs}

For signals $\mathbf{f}, \mathbf{g} \in L^{2}(\mathbb{R})$, the convolution product $\mathbf{h}=\mathbf{f} * \mathbf{g}$ satisfies

$$
\mathbf{h}=(\mathbf{f} * \mathbf{g})=\int_{\mathbb{R}} \hat{\mathbf{h}}(\xi) \exp \{2 \pi i \xi t\} d \xi=\int_{\mathbb{R}} \hat{\mathbf{f}}(\xi) \hat{\mathbf{g}}(\xi) \exp \{2 \pi i \xi t\} d \xi .
$$


By replacing the complex exponentials in (5) with the graph Fourier transform, i.e., the graph Laplacian eigenvectors $\Lambda_{l}$, in [14 has defined a generalised convolution of signals $\mathbf{f}, \mathbf{g} \in \mathrm{V}$ by

$$
\begin{array}{r}
(\mathbf{f} * \mathbf{g}):=\sum_{l=1}^{N}\left[\hat{\mathbf{f}}(l) \hat{\mathbf{g}}(l) \Lambda_{l}\right]=\sum_{l=1}^{N}\left[\left(\sum_{k=1}^{N} \Lambda_{l}^{*}(k) \mathbf{f}(k)\right)\left(\sum_{k=1}^{N} \Lambda_{l}^{*}(k) \mathbf{g}(k)\right) \Lambda_{l}\right] \\
\sum_{l=1}^{N}\left[\left(\Lambda_{l}^{*} \mathbf{f}\right)\left(\Lambda_{l}^{*} \mathbf{g}\right) \Lambda_{l}\right]=\sum_{l=1}^{N}\left[\Lambda_{l}^{*}(\mathbf{f g}) \Lambda_{l}\right]=\sum_{l=1}^{N} \mathbf{f}(l) \mathbf{g}(l) .
\end{array}
$$

Proposition 2. The linear operator associated to the convolution signal function $\mathbf{g}$ commutes with the stacking of cross-sections according to the thresholdlinear superposition,i.e.,

$$
(\mathbf{f} * \mathbf{g})=\int_{0}^{+\infty}(\chi(\mathbf{f}, \lambda) * \mathbf{g}) d \lambda
$$

\subsection{Diffusion on graphs}

Consider an arbitrary graph $\mathcal{G}=(\mathcal{V}, E, \mathbf{W})$ with Laplacian matrix $\mathbf{L}$ and a signal $\mathbf{f} \in \mathcal{V} \rightarrow \mathbb{R}^{N}$. For a given constant $\sigma>0$, define the time-varying vector $\mathbf{f}_{\sigma, t} \in \mathbb{R}^{N}$ as the solution of the linear differential equation:

$$
\frac{\partial \mathbf{f}_{\sigma, t}}{\partial t}=-\sigma \mathbf{L} \mathbf{f}_{\sigma, t}, \quad \mathbf{f}_{0}=\mathbf{f}
$$

where $\sigma$ is the thermal conductivity [16] and controls the heat diffusion rate. The differential equation in (7) represents the heat diffusion process on the graph $\mathcal{G}$ due to the fact that $-\mathbf{L}$ can be shown to be the discrete approximation of the continuous Laplacian operator used to characterise the heat diffusion in physics [1624]. The general solution of the heat equation on $\mathbb{R}^{N}$ is obtained by convolution [25. However, the solution of (7) denoted by $\mathbf{f}_{\sigma, t}^{\mathrm{L}} \in \mathrm{V} \times \mathrm{V}$, is given by the matrix exponential as follows

$$
\mathbf{f}_{\sigma, t}^{\mathbf{L}}:=\exp (-\sigma \mathbf{L} t) \mathbf{f}
$$

which can be verified by direct substitution in (7). It is important to note that for a given time $t$, the $n$-th element of $\mathbf{f}_{\sigma, t}(n)$ is

$$
\frac{\partial \mathbf{f}_{\sigma, t}^{\mathbf{L}}(n)}{\partial t}=\sum_{k \in \mathcal{N}(n)} \sigma \mathbf{W}(n, k)\left(\mathbf{f}_{\sigma, t}^{\mathbf{L}}(k)-\mathbf{f}_{\sigma, t}^{\mathbf{L}}(n)\right)
$$

where $\mathcal{N}(n)$ is the neighbourhood of $n$, i.e., the $k$ such that $\mathbf{W}(i, k)>0$. Thus, the heat flow on an edge grows proportionally with both the "temperature differential" $\mathbf{f}_{\sigma, t}^{\mathbf{L}}(k)-\mathbf{f}_{\sigma, t}^{\mathbf{L}}(n)$ and the weight $\mathbf{W}(n, k)$. Now in Prop. 3 , we see the behaviour of the graph diffusion in the stack of cross-sections. 
Proposition 3. The operator $\mathbf{f}_{\sigma, t}^{\mathbf{L}}$ in eq. (8) associated to the diffusion of a graph signal $\mathbf{f}$, commutes with the stacking of cross-sections according to the threshold-linear superposition, i.e.,

$$
\mathbf{f}_{\sigma, t}^{\mathbf{L}}(n)=\int_{0}^{+\infty}(\chi(\mathbf{f}, \lambda))_{\sigma, t}^{\mathbf{L}}(n) d \lambda
$$

Note that the right part of the equality means that the graph diffusion is applied in each upper level set of the graph signal function $\mathbf{f}$. The proof of Prop. 3 is straightforward by means of Taylor series expansion of the graph heat equation and interchanging summation and integration.

Proof.

$\mathbf{f}_{\sigma, t}^{\mathbf{L}}(n)=\sum_{k=0}^{\infty} \frac{(-\sigma \mathbf{L} t)^{k} \mathbf{f}}{k !}=\int_{0}^{+\infty} \sum_{k=0}^{\infty} \frac{(-\sigma \mathbf{L} t)^{k}}{k !} \chi(\mathbf{f}, \lambda)(n) d \lambda=\int_{0}^{+\infty}(\chi(\mathbf{f}, \lambda))_{\sigma, t}^{\mathbf{L}}(n) d \lambda$.

At this point, we should highlight that the behaviour of the diffusion in $(8)$ is controlled by the choice of the Laplacian matrix, i.e, therefore expression (8) includes isotropic and anisotropic diffusion.

\subsection{Morphological operators in graphs}

In the case of a graph value function $\mathbf{f} \in \mathrm{V}$, we can have the following counterparts of dilation and erosion of numerical functions [26|27|28] viewed as a convolution in max-plus algebra (and its adjoint/dual algebra).

Definition 6. The matrix $\mathbf{W}$ is a morphological weight matrix if $-\infty \leq \mathbf{W}(n, m) \leq$ 0 , for all $n, m$.

It is important to note, that it is a really simple characterisation of the weight matrix because we do not require symmetry $(\mathbf{W}(n, m) \neq \mathbf{W}(m, n))$ neither zero-diagonal $(\mathbf{W}(i, i) \neq 0)$.

Definition 7. The dilation of a signal function $\mathbf{f}$ on a graph $\mathcal{G}=(\mathcal{V}, E)$ is defined by

$$
\delta_{\mathbf{W}}(\mathbf{f})(n)=\bigvee_{m=1}^{N}(\mathbf{f}(m)+\mathbf{W}(n, m):=\mathbf{W} \oplus \mathbf{f}(n)
$$

and the dual adjoint erosion is given by

$$
\varepsilon_{\mathbf{W}} \mathbf{f}(n):=\bigwedge_{m=1}^{N}\left(\mathbf{f}(m)+\mathbf{W}^{*}(n, m)\right)=\bigwedge_{m=1}^{N}(\mathbf{f}(m)-\mathbf{W}(m, n)):=\mathbf{W}^{*} \ominus \mathbf{f}(n)
$$

We remark that, $\varepsilon_{\mathbf{W}}, \delta_{\mathbf{W}}$ are both in $\mathrm{V} \times \mathrm{V}$ and include morphological transformations by flat, non-flat [26 27, adaptive [28] and nonlocal structuring elements [29]. The following proposition list include some properties easy to check. 
Proposition 4. Let $\mathbf{f}_{i} \in \mathrm{V}, i=1, \ldots, k$ be signal on graph and let $\mathbf{W}_{j}, j=$ $1, \ldots m$ be morphological weights matrices:
1. $\mathbf{W}_{m} \oplus \ldots \oplus \mathbf{W}_{1} \oplus(\mathbf{f})=\left(\sum_{j=1}^{m} \mathbf{W}_{j}\right) \oplus \mathbf{f}$
2. $\mathbf{W} \oplus\left(\bigvee_{i=1}^{k} \mathbf{f}_{i}\right)=\bigvee_{i=1}^{k}\left(\mathbf{W} \oplus \mathbf{f}_{i}\right)$
3. $\mathbf{W}_{m}^{*} \ominus \ldots \ominus \mathbf{W}_{1}^{*} \ominus \mathbf{f}=\left(\sum_{j=1}^{m} \mathbf{W}_{j}^{*}\right) \ominus \mathbf{f}$
4. $\mathbf{W}^{*} \ominus\left(\bigwedge_{i=1}^{k} \mathbf{f}_{i}\right)=\bigwedge_{i=1}^{k}\left(\mathbf{W}^{*} \ominus \mathbf{f}_{i}\right)$
5. For $\mathbf{f} \leq \mathbf{g}$ then $\mathbf{W} \oplus \mathbf{f} \leq \mathbf{W} \oplus \mathbf{g}$
6. For $\mathbf{f} \leq \mathbf{g}$ then $\mathbf{W}^{*} \ominus \mathbf{f} \leq \mathbf{W}^{*} \ominus \mathbf{g}$
7. $\mathbf{W}^{*} \ominus(\mathbf{W} \oplus \mathbf{f}) \geq \mathbf{f} \geq \mathbf{W} \oplus\left(\mathbf{W}^{*} \ominus \mathbf{f}\right)$

A crucial point is the existence of a Galois adjunction theorem [30]31 for graph valued signals.

Theorem 1. Given a $\mathbf{W}$ morphological weight matrix and, the pair of operators $\left(\varepsilon_{\mathbf{W}}, \delta_{\mathbf{W}}\right)$, defines an Galois adjunction, i.e., for all $\mathbf{f}, \mathbf{g} \in \mathbf{V}$, we have $\mathbf{W} \oplus \mathbf{f} \leq$ $\mathbf{g} \Longleftrightarrow \mathbf{f} \leq \mathbf{W}^{*} \ominus \mathbf{g}$.

Proof.

$$
\begin{gathered}
\mathbf{W} \oplus \mathbf{f} \leq \mathbf{g} \Longleftrightarrow \forall n, \quad \bigvee_{m=1}^{N}(\mathbf{f}(m)+\mathbf{W}(n, m)) \leq \mathbf{g}(n) \\
\Longleftrightarrow \forall n, \forall m, \mathbf{f}(m)+\mathbf{W}(n, m) \leq \mathbf{g}(n), \Longleftrightarrow \forall n, \forall m, \mathbf{f}(m) \leq \mathbf{g}(n)-\mathbf{W}(n, m), \\
\Longleftrightarrow \forall m, \mathbf{f}(m) \leq \bigwedge_{n=1}^{N} \mathbf{g}(n)-\mathbf{W}(n, m), \Longleftrightarrow \forall m, \mathbf{f}(m) \leq \bigwedge_{n=1}^{N} \mathbf{g}(n)+\mathbf{W}^{*}(m, n), \\
\Longleftrightarrow \forall m, \mathbf{f} \leq \mathbf{W}^{*} \ominus \mathbf{g} .
\end{gathered}
$$

However, we do not have an order between the original signal $\mathbf{f}$ and its dilation or erosion, i.e., we have $\mathbf{W}^{*} \ominus \mathbf{f} \leq \mathbf{W} \oplus \mathbf{f}$, but $\mathbf{f} \not \leq \mathbf{W} \oplus \mathbf{f}$ neither $\mathbf{W} \ominus \not \leq \mathbf{f}$.

Definition 8. A morphological weight matrix $\mathbf{W}$ is called conservative if $\mathbf{W}(i, i)=$ 0 for all $i \in 1 \ldots, N$.

Proposition 5. If $\mathbf{W}$ is an extensive morphological weight matrix then $\mathbf{W}^{*} \ominus$ $\mathbf{f} \leq \mathbf{f} \leq \mathbf{W} \oplus \mathbf{f}$ for every $\mathbf{f}$.

Thanks to theorem 1, we can have a large set of morphological filters such as openings, closings, alternate sequential filters, levelling and so on, because they are are defined by combination of dilations and erosions [32 33].

Definition 9. A morphological weight matrix $\mathbf{B}$ is called flat if it is morphological weight matrix and $\mathbf{B}(i, j)=0$ or $\mathbf{B}(i, j)=-\infty$ for all $i, j \in 1 \ldots, N$. 
Proposition 6. The flat dilation and erosion obey both the threshold-max superposition and the threshold linear superposition, i.e.,

$$
\begin{aligned}
& \delta_{\mathbf{B}}(\mathbf{f})=\bigvee_{\lambda=0}^{+\infty}\left\{\delta_{\mathbf{B}}(\chi(\mathbf{f}, \lambda))=1\right\}=\int_{0}^{+\infty} \delta_{\mathbf{B}}(\chi(\mathbf{f}, \lambda)) d \lambda \\
& \varepsilon_{\mathbf{B}}(\mathbf{f})=\bigvee_{\lambda=0}^{+\infty}\left\{\varepsilon_{\mathbf{B}}(\chi(\mathbf{f}, \lambda))=1\right\}=\int_{0}^{+\infty} \varepsilon_{\mathbf{B}}(\chi(\mathbf{f}, \lambda)) d \lambda
\end{aligned}
$$

Since by Props. 1 and 6, we can directly have that the class of operators $\phi \in \mathrm{V} \times \mathrm{V}$ that obey the threshold-linear superposition also contain morphological gradients (difference between dilation and erosion), opening and closing (composition of dilation and erosion), top-hat transformation, granulometries, reconstruction operators, levelling, additive morphological decompositions 34] and skeleton transformation (based on generalised Lantuejoul formula) 35.

\subsection{Morphological operators via convolution on graph}

Definition 10. For a graph signal value $\mathbf{f} \in \mathrm{V}$, the convolution-thresholding nonlinear operator associated to the heat diffusion $\mathbf{W}$ of conductivity $\sigma$ at scale $t$, and the threshold $\tau$, with $\tau \in[0,1]$, is the mapping $\mathcal{F}(\mathcal{V}, \mathbb{R}) \rightarrow \mathcal{F}(\mathcal{V}, \mathbb{R})$ defined by

$$
\psi_{\mathbf{L}, \tau}(\mathbf{f})=\int_{0}^{+\infty}\left[(\chi(\mathbf{f}, \lambda))_{\sigma, t}^{\mathbf{L}} \geq \tau\right] d \lambda
$$

The next proposition is easy to proof.

Proposition 7. For all $\mathbf{f} \in \mathbf{V}:$ a) $\psi_{\mathbf{L}, \tau}(\mathbf{f})$ satisfies the threshold-linear superposition in Def. 2. b) $\psi_{\mathbf{L}, \tau}(\mathbf{f})$ is monotonous with respect to the choice of $\tau$, i.e, $\tau_{1} \leq \tau_{2} \Rightarrow \psi_{\mathbf{L}, \tau_{1}}(\mathbf{f}) \leq \psi_{\mathbf{L}, \tau_{2}}(\mathbf{f})$.

We can also prove that 12 is increasing.

Proposition 8. If $\mathbf{f}_{1} \leq \mathbf{f}_{2}$, then $\psi_{\mathbf{L}, \tau}\left(\mathbf{f}_{1}\right) \leq \psi_{\mathbf{L}, \tau}\left(\mathbf{f}_{2}\right)$ for all $\sigma, t \geq 0$.

Proof. We note that (8) follows the called comparison principle, (Lemma 2.6, property (d) in [17]), i.e. if $\mathbf{f}_{1} \leq \mathbf{f}_{2}$, then $\left(\mathbf{f}_{1}\right)_{\sigma, t}^{\mathbf{L}} \leq\left(\mathbf{f}_{2}\right)_{\sigma, t}^{\mathbf{L}}$ for all $\sigma, t \geq 0$. The proposition is proved by applying this result in each upper level set and integrating in $\lambda$.

Proposition 9. For the case of a flat morphological weight matrix $\mathbf{B}$, the morphological flat operators in Prop. 6 correspond to the convolution-thresholding nonlinear operator in 12 with particular values of $\tau$ as follows: $\delta_{\mathbf{B}}(\mathbf{f})=\lim _{\tau \rightarrow 0^{+}} \psi_{\mathbf{B}, \tau}(\mathbf{f})$ and $\varepsilon_{\mathbf{B}}(\mathbf{f})=\lim _{\tau \rightarrow 1^{-}} \psi_{\mathbf{B}, \tau}(\mathbf{f})$. 
Proposition 10. For a binary signal $S \in \mathcal{V} \times\{0,1\}$, the set of measures $\operatorname{Vol}(S)=$ $\sum_{i \in S} d_{i}$. Let $\rho(\mathbf{L})$ be the spectral radius of the graph Laplacian, $\mathbf{L}$, then iterations in Prop. 9 on the graph with initial set $S$ are stationary if either of the two conditions are satisfied:

$$
\sigma \leq \rho(\mathbf{L})^{-1} \log \left(1+\tau d_{-}^{r / 2}(\operatorname{Vol}(S))^{-1 / 2}\right) \text { or } \sigma \leq \frac{\tau}{\|\mathbf{L} \chi(S)\|_{\mathrm{V}, \infty}}
$$

The proof is direct by using Lemma 2.2 and Theorem 4.2 in [17.

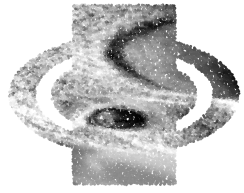

(a) Original

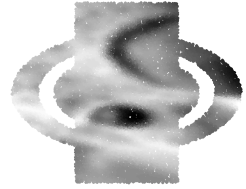

(b) Diffusion $(t=10, \sigma=.02)$

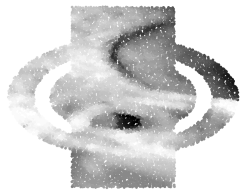

(e) $\tau=0.2$

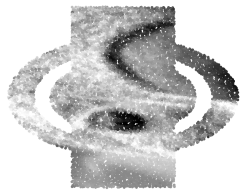

(h) $\tau=0.2$

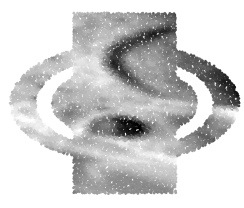

(f) $\tau=0.5$

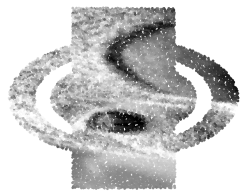

(i) $\tau=0.5$

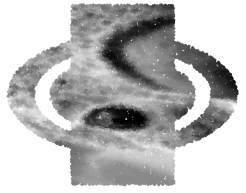

(c) Non-flat erosion

(d) Non-flat dilation

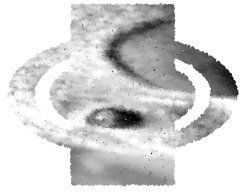

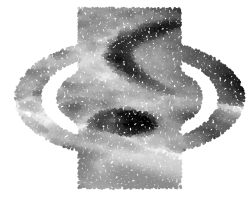

(g) $\tau=0.8$

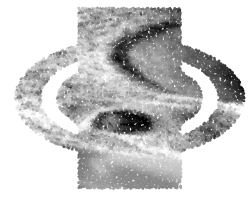

(j) $\tau=0.8$

Fig. 1. Family of linear (b) and nonlinear transformations via thresholding of diffusion process. Second row: $(\sigma=.1, t=10)$. Third row: $(\sigma=.01, t=10)$

Now, we point out a link of the operator in 12 with motion by mean curvature on a graph.

Proposition 11. The operator in $(12)$ in the case of $\tau=.5$ is an iteration with of the approximate motion by mean curvature on a graph.

Firstly, the $\psi_{\mathbf{L}, .5}$ is an iteration of the well-known Merriman, Bence and Osher (MBO) [37] 38] threshold dynamics algorithms on graphs. The $M B O$ algorithm is obtained by time splitting the Allen-Cahn phase-field equation for motion 


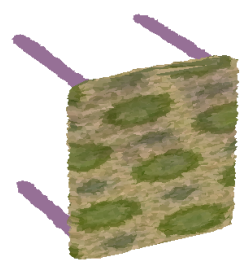

(a) Original

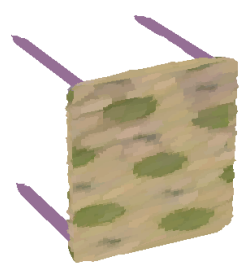

(b) Dilation

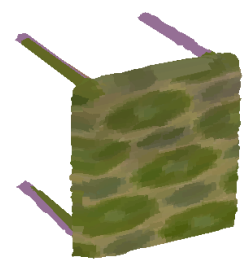

(c) Erosion

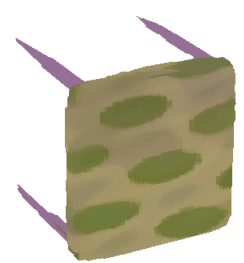

(d) Curvature motion

Fig. 2. Illustration of nonlinear filters on textured mesh. The textured mesh is obtained from 36. Note that both colours and mesh coordinates change in the processing. (d) Curvature motion is obtained by iterating $\psi_{\mathbf{L}, .5}$.

by mean curvature 2 The resulting scheme alternated two steps, diffusion, and simple thresholding [17]. Secondly, several papers use the MBO algorithm on a graph to approximate motion by mean curvature [17/40. It is important to note that the curvature is defined by means of the isotropic total variation [17] instead of the one-Laplacian as it is the case in [1011]12 13.

\section{Experimental section}

The family of filters proposed can be used to analyse any function defined on the vertices of a graph. We provide some illustrations of the results in grey scale functions on graph in Fig. 11 colour data on mesh in Fig.2 and images on a graph of images in Fig. 3. We first constructed a graph from these datasets by treating the nodes in the graph to be the sample points in the dataset and the edges weight to be the similarity between the features of the different samples. Edge weights were determined via the Radial Bases Function (RBF) kernel with $\sigma^{2}$ set to the variance in the respective dataset $\mathbf{W}(i, j)=\exp \left(-\frac{\left\|\mathbf{x}_{i}-\mathbf{x}_{j}\right\|^{2}}{2 \sigma^{2}}\right)$. Finally, in Fig. 3 we have considered a subset of the USPS handwritten digit database for illustration. Each image is a digit in a $28 \times 28$ grey scale image which is considered in our approach as a multivariate vector in $\mathbb{R}^{784}$. This random weight

${ }^{2}$ The semilinear heat equation called the Allen-Cahn equation is a reaction-diffusion equation of mathematical physics of the form:

$$
\frac{\partial u}{\partial t}-\delta u+\frac{W^{\prime}(u)}{\epsilon^{2}}=0 \in \mathbb{R}^{N} \times(0, \infty)
$$

which was introduced by S.M. Allen and J.W. Can (1979) 39 to describe the process of phase separation in iron alloys, including order-disorder transitions. Here $W$ is a function that has only two equal minima; its typical form is $W(v)=\frac{\left(v^{2}-1\right)^{2}}{2}, W^{\prime}$ denotes the derivative and $\epsilon$ is a positive parameter. 
graph has nodes on images and weights in the 5-KNN graph considering the Euclidean distance. We only use 200 images of two digits $(0,9)$ to illustrated the merits of our approach and its potential applications.

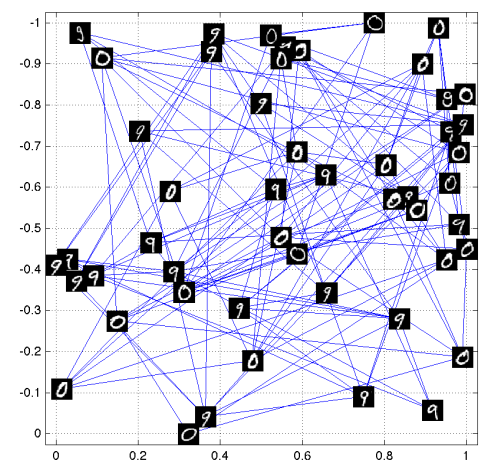

(a) Original

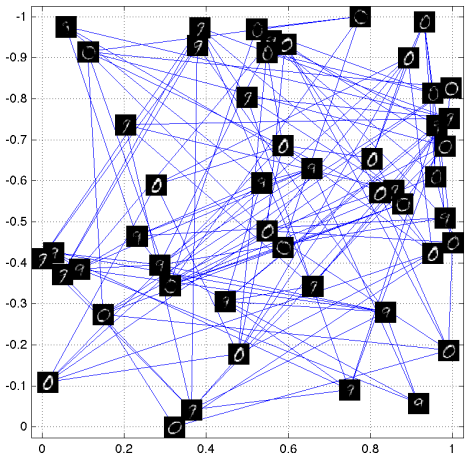

(c) $\tau=.1$

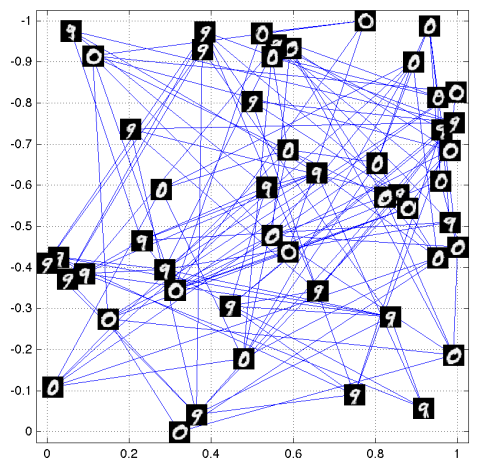

(b) $\tau=.9$

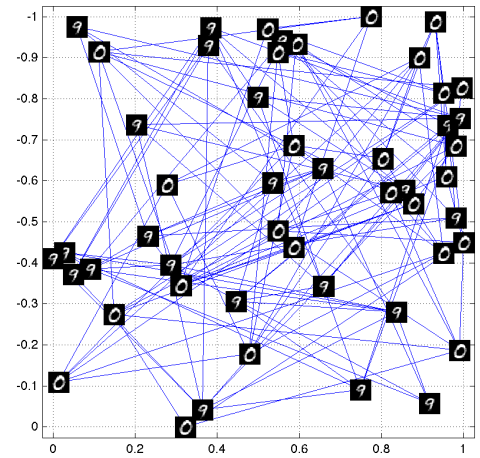

(d) $\tau=.5$ after 10 iterations.

Fig. 3. $\mathcal{G}$ is the 5 nearest neighbours with $\mathbf{W}$ the Euclidean distance between pairs of images. Lines are linking images that where $\mathbf{W}(i, j)$ is not zero. The parameter in the diffusion are $t=20$ and $\sigma=.03$. Note that digits in the same class tend to be similar in (d). 


\section{Conclusion and perspectives}

We have analysed nonlinear operators on stack of graphs as a discrete adaptation of non-flat morphological transformation. This approach is based on connection between diffusion+ thresholding operators and morphological operators. We have proved adjunction of pair dilation and erosion in signal graphs in a general case. Finally, we have illustrated the interest and behaviour of such operators in some problems of image processing and pattern recognition.

\section{References}

1. D. I. Shuman, S. K. Narang, P. Frossard, A. Ortega, and P. Vandergheynst, "The emerging field of signal processing on graphs: Extending high-dimensional data analysis to networks and other irregular domains," S. P. Magazine, IEEE, vol. 30, no. 3, pp. 83-98, 2013.

2. S. Calderon and T. Boubekeur, "Point morphology," ACM Transactions on Graphics (TOG), vol. 33, no. 4, p. 45, 2014.

3. L. Vincent, "Graphs and mathematical morphology," S. P., vol. 16, no. 4, pp. 365-388, 1989.

4. H. Heijmans, P. Nacken, A. Toet, and L. Vincent, "Graph morphology," Journal of Visual Communication and Image Representation, vol. 3, no. 1, pp. 24-38, 1992.

5. J. Cousty, L. Najman, F. Dias, and J. Serra, "Morphological filtering on graphs," Computer Vision and Image Understanding, vol. 117, no. 4, pp. 370-385, 2013.

6. L. Najman and J. Cousty, "A graph-based mathematical morphology reader," Pattern Recognition Letters, vol. 47, no. 1, pp. 3-17, Oct 2014.

7. F. Meyer, "Watersheds on weighted graphs," Pattern Recognition Letters, vol. 47, no. 1, pp. 72-79, Oct 2014.

8. L. Najman, F. Meyer et al., "A short tour of mathematical morphology on edge and vertex weighted graphs," Image Processing and Analysis with Graphs: Theory and Practice, pp. 141-174, 2012.

9. I. Bloch and A. Bretto, "Mathematical morphology on hypergraphs: Preliminary definitions and results," in Dis. Geom. for Comp. Imag., 2011, pp. 429-440.

10. A. Elmoataz, O. Lezoray, and S. Bougleux, "Nonlocal discrete regularization on weighted graphs: a framework for image and manifold processing," Trans. Im. Proc., vol. 17, no. 7, pp. 1047-1060, 2008.

11. V.-T. Ta, A. Elmoataz, and O. Lézoray, "Partial difference equations over graphs: Morphological processing of arbitrary discrete data," in Computer Vision-ECCV 2008. Springer, 2008, pp. 668-680.

12. — - "Nonlocal pdes-based morphology on weighted graphs for image and data processing," Trans. Im. Proc., vol. 20, no. 6, pp. 1504-1516, 2011.

13. A. Elmoataz, X. Desquesnes, O. Lezoray et al., "Non-local morphological PDEs and $p$-Laplacian equation on graphs with applications in image processing and machine learning," J. of Sel.Top. in S. P., vol. 6, no. 7, pp. 764-779, 2012.

14. D. I. Shuman, B. Ricaud, and P. Vandergheynst, "A windowed graph fourier transform," in Statistical S.P. Workshop (SSP), 2012 IEEE, 2012, pp. 133-136.

15. A. Sandryhaila and J. Moura, "Big data analysis with signal processing on graphs: Representation and processing of massive data sets with irregular structure," $S$. P. Magazine, IEEE, vol. 31, no. 5, pp. 80-90, Sept 2014. 
16. S. Segarra, W. Huang, and A. Ribeiro, "Diffusion and superposition distances for signals supported on networks," CoRR, vol. abs/1411.7443, 2014.

17. Y. van Gennip, N. Guillen, B. Osting, and A. L. Bertozzi, "Mean curvature, threshold dynamics, and phase field theory on finite graphs," Milan Journal of Mathematics, vol. 82, no. 1, pp. 3-65, 2014.

18. A. E. Chakik, A. Elmoataz, and X. Desquesnes, "Mean curvature flow on graphs for image and manifold restoration and enh." S. P., vol. 105, pp. 449 - 463, 2014.

19. C. Garcia-Cardona, E. Merkurjev, A. L. Bertozzi, A. Flenner, and A. Percus, "Fast multiclass segmentation using diffuse interface methods on graphs," arXiv preprint arXiv:1302.3913, 2013.

20. F. R. K. Chung, Spectral Graph Theory (CBMS Regional Conference Series in Mathematics, No. 92). American Mathematical Society, Dec. 1996.

21. P. Wendt, E. J. Coyle, and N. C. Gallagher Jr, "Stack filters," Ac., Sp. and S. P., IEEE Trans. on, vol. 34, no. 4, pp. 898-911, 1986.

22. P. Maragos and R. W. Schafer, "Morphological filters-part ii: Their relations to median, order-statistic, and stack filters," Ac., Sp. and S. P., IEEE Trans. on, vol. 35, no. 8, pp. 1170-1184, 1987.

23. P. Maragos and R. D. Ziff, "Threshold superposition in morphological image analysis systems," Trans. Patt. Ana. Mach. Lear., vol. 12, no. 5, pp. 498-504, 1990.

24. R. I. Kondor and J. Lafferty, "Diffusion kernels on graphs and other discrete input spaces," in ICML, 2002, pp. 315-322.

25. L. C. Evans, Partial differential equations. Providence, Rhode Land: American Mathematical Society, 1998.

26. H. Heijmans, "Theoretical aspects of gray-level morphology," Trans. Patt. Ana. Mach. Lear., vol. 13, no. 6, pp. 568-582, 1991.

27. P. Soille, Morphological Image Analysis. Springer-Verlag, 1999.

28. V. Ćurić, A. Landström, M. J. Thurley, and C. L. Luengo Hendriks, "Adaptive mathematical morphology-a survey of the field," Pat. Rec. Letters, 2014.

29. S. Velasco-Forero and J. Angulo, "On nonlocal mathematical morphology," in Math. Morph. and Its App. to Signal and I. P. Springer, 2013, pp. 219-230.

30. H. J. Heijmans and C. Ronse, "The algebraic basis of mathematical morphology i. dilations and erosions," CVGIP, vol. 50, no. 3, pp. 245-295, 1990.

31. C. Ronse and H. J. Heijmans, "The algebraic basis of mathematical morphology: Ii. openings and closings," CVGIP, vol. 54, no. 1, pp. 74-97, 1991.

32. P. Maragos, "Lattice image processing: a unification of morphological and fuzzy algebraic systems," J. of Math. Im. and Vis., vol. 22, no. 2-3, pp. 333-353, 2005.

33. C. Ronse, "Why mathematical morphology needs complete lattices," S.P., vol. 21, no. 2, pp. 129-154, 1990.

34. S. Velasco-Forero and J. Angulo, "Classification of hyperspectral images by tensor modeling and additive morph. decom." Pat. Rec., vol. 46, no. 2, pp. 566-577, 2013.

35. L. Najman and H. Talbot, Mathematical Morphology. John Wiley \& Sons, 2013.

36. S. e. a. Biasotti, "SHREC 14 track: Retrieval and classification on textured 3d models," Eurographics Workshop on 3D Object Retrieval, pp. 111-120, 2014.

37. B. Merriman, J. K. Bence, and S. J. Osher, "Motion of multiple junctions: A level set approach," Journal of Comp. Physics, vol. 112, no. 2, pp. 334-363, 1994.

38. — Diffusion generated motion by mean curvature. Elsevier, 1992.

39. S. M. Allen and J. W. Cahn, "A microscopic theory for antiphase boundary motion and its application to antiphase domain coarsening," Acta Metallurgica, vol. 27, no. 6, pp. 1085-1095, 1979.

40. E. Merkurjev, T. Kostic, and A. L. Bertozzi, "An MBO scheme on graphs for segmentation and image proc." SIAM J. I. S., vol. 6, no. 4, pp. 1903-1930, 2013. 